\title{
A CLASSIFICAÇÃO FUNCIONAL NO ESPORTE PARALÍMPICO
}

\author{
Vinicius Denardin Cardoso, Universidade Federal do Rio Grande do Sul - UFRGS - \\ Porto Alegre, Rio Grande do Sul-Brasil \\ Adroaldo Cesar Gaya, Universidade Federal do Rio Grande do Sul-UFRGS - Porto \\ Alegre, Rio Grande do Sul - Brasil
}

\section{RESUMO}

Os sistemas de classificação funcional têm sido utilizados nos esportes paralímpicos, a fim de estabelecer um ponto de partida justo e igualitário para as competições. A classificação funcional surgiu com o objetivo de assegurar a competição justa e eliminar as possibilidades de injustiças entre participantes de classes semelhantes. O objetivo deste estudo é realizar uma revisão na literatura referente à classificação funcional no esporte paralímpico e de como é utilizada nos diferentes tipos de deficiência. A revisão aborda estudos publicados entre os anos de 1990 até 2013, obtidos em bancos de dados eletrônicos: Medline, Academic Search, ScienceDirect e SportDiscus e busca manual em livros sobre a temática. Foram incluídos na revisão apenas os textos publicados em Inglês e Português. O aprimoramento dos diferentes sistemas de classificação funcional é constante, esta continua evolução é fundamental para garantir que o nível de treinamento e a habilidade de cada indivíduo sejam os fatores decisivos para o sucesso no esporte paralímpico.

Palavras-Chave: Classificação funcional; Esporte paralímpico; Sistemas de Classificação Funcional.

\section{FUNCTIONAL CLASSIFICATION IN PARALYMPIC SPORTS}

\begin{abstract}
The functional classification begin with the aim of ensuring fair competition and eliminate the possibilities of injustices among participants of similar classes. The aim of this study is to conduct a review in the literature concerning the functional classification in Paralympic sport and how it is used in different types of disabilities. The review discusses studies published between the years of 1990 until 2013, obtained in electronic databases: Medline, Academic Search, ScienceDirect and SportDiscus and manual search in books on the subject. Were included in the review only the texts published in English and Portuguese. The improvement of different functional classification systems is constant, this continues evolution is essential to ensure that the level of training and the ability of each individual are the deciding factors for success in Paralympic sport.
\end{abstract}

Key-Words: Functional classification; Paralympic sport; Functional Classification Systems. 


\section{LA CLASIFICACIÓN FUNCIONAL EN EL DEPORTE PARALÍMPICO}

\section{RESUMEN}

Los sistemas de clasificación funcional han utilizado en deportes Paralímpicos, con el fin de establecer un punto de partida justo y equitativo para las competiciones. La clasificación funcional surgió con el objetivo de garantizar una competencia leal y eliminar las posibilidades de las injusticias entre los participantes de clases similares. El objetivo de este estudio es realizar una revisión de la literatura referente a la clasificación funcional en el deporte paralímpico y cómo se utiliza en diferentes tipos de discapacidad. La revisión analiza los estudios publicados entre los años 1990 hasta 2013, obtenidos en bases de datos electrónicas: Medline, Academic Search, ScienceDirect y SportDiscus y búsqueda manual en libros sobre el tema. Se incluyeron en la revisión sólo los textos publicados en inglés y portugués. La mejora de los sistemas de clasificación funcional diferente es constante, esta continúa evolución es fundamental para asegurar que el nivel de formación y la capacidad de cada individuo son los factores decisivos para el éxito en el deporte paralímpico.

Palabras-Clave: Clasificación funcional; Deporte paralímpico; Sistemas de Clasificación Funcional. 


\section{INTRODUÇÃO}

O Esporte Adaptado surgiu como um importante meio na reabilitação física, psicológica e social para pessoas com algum tipo de deficiência, são adaptações e modificações em regras, materiais e espaços, que possibilitam a participação de pessoas com deficiência dentro das modalidades desportivas. Também pode ser definido como esporte modificado ou especialmente criado para ir ao encontro das necessidades únicas de indivíduos com algum tipo de deficiência. ${ }^{1}$

A oportunidade da prática esportiva para pessoas com deficiência é de grande eficácia para a promoção da qualidade de vida das mesmas, o esporte é uma oportunidade de testar as potencialidades e possibilidades de atletas com deficiência, encorajando para buscar integração. ${ }^{2}$

Com o tempo, o esporte adaptado foi ganhando muitos adeptos, novas modalidades foram surgindo, diferentes tipos de deficiências ganharam espaço dentro das práticas esportivas e consequentemente a competição ficou evidente no esporte adaptado. Dessa forma, é considerada a nomenclatura do esporte competitivo para pessoas com deficiência como Esporte Paralímpico.

Dessa forma a classificação funcional surgiu no Esporte Paralímpico com o objetivo de assegurar a legítima participação de atletas com deficiências, independente da natureza e o grau da deficiência, ${ }^{3}$ assim a classificação utilizada no esporte paralímpico constitui-se em um nivelamento entre os aspectos da capacidade física e competitiva, colocando as deficiências semelhantes em um grupo determinado. ${ }^{4}$

Isso permite igualar a competição entre indivíduos com várias seqüelas de deficiência, pois o sistema de classificação eficiente é o pré-requisito para uma competição mais equiparada. ${ }^{5-6}$

O surgimento de um sistema de classificação funcional foi de crucial importância nas diversas modalidades paralímpicas, e se tornou um fator de nivelamento no que diz respeito aos aspectos competitivos, garantindo direitos e condições de igualdade e minimizando injustiças dentro do desporto paralímpico. ${ }^{7-8}$ 
A classificação funcional é considerada como a essência do esporte paraolímpico ${ }^{9}$ e tem se aprimorado ao longo da evolução do movimento paralímpico. Durante essa evolução, muitas alterações ocorreram no processo de classificação em diferentes deficiências e modalidades esportivas, dessa forma, com objetivo de proporcionar informações atuais, este estudo realiza uma revisão de literatura sobre a classificação funcional no esporte paralímpico.

\section{METODOLOGIA}

O presente estudo se caracteriza por ser uma revisão da literatura baseada em referências nacionais e internacionais obtidas em bancos de dados eletrônicos: Medline, Academic Search, Sciencedirect e Sportdiscus e busca manual em livros sobre a temática. Foram incluídos na revisão apenas os textos publicados em Inglês e Português entre os anos de 1990 a 2013, que apresentavam como descritores os termos: classificação funcional, classificação funcional no esporte paralímpico e sistemas de classificação funcional e ainda functional classification; functional classification in paralympic sports e functional classification system.

\section{ORIGEM DO ESPORTE PARALÍMPICO}

A prática esportiva para pessoas com deficiência física na Alemanha teria iniciado em 1918, para amenizar os horrores da Primeira Guerra Mundial ${ }^{10}$ e em 1932 em Glasgow, no Reino Unido foi formada uma associação de jogadores de golfe, abarcando amputados unilaterais de membros superiores. ${ }^{11}$

O término da Segunda Guerra Mundial (1939-1945) foi um marco para a evolução do esporte adaptado para pessoas com deficiência, pois muitos soldados retornavam aos seus países e traziam em seu corpo marcas que jamais esqueceriam. O pós-guerra deixou muitos soldados mutilados, com distúrbios motores, visuais e auditivos, isso fez com que seus governos tomassem uma série de providências sobre a qualidade de vida desses indivíduos. Com isso muitos começaram a ter acesso às práticas esportivas e atividades físicas adaptadas como forma de minimizar as adversidades causadas pela guerra. 
Em Aylesbury na Inglaterra, o médico neurologista alemão Ludwig Gutmann e seus colegas do Stoke Mandeville Hospital obtiveram inúmeros sucessos com a reabilitação de ex-combatentes através de jogos esportivos com cadeira de rodas. ${ }^{1,11-12}$

Em 1948 aconteceram os Jogos Olímpicos de Verão, em Londres, Inglaterra, e Ludwig Gutmann, aproveita o evento e cria paralelamente os primeiros jogos de Stoke Mandeville para paraplégicos, estes jogos contaram com a participação de 16 atletas ingleses nas modalidades de: arco e flecha, tiro ao alvo e arremesso de dardo. ${ }^{13-15}$

Já em 1960, em Roma na Itália foram realizados os Jogos de Stoke Mandeville, logo após o encerramento dos Jogos Olímpicos. Este evento teve a participação de 230 atletas de 23 países, contou com o apoio do Comitê Olímpico Italiano (COI), tendo como madrinha dos jogos a primeira dama italiana, Sra. Carla Grounchi. Este evento marca o envolvimento político e social do mundo todo com os jogos para pessoas com deficiência. ${ }^{11,13}$

No Brasil, a história do esporte paralímpico surge em 1958, quando surgiram dois clubes de basquete em cadeira de rodas, em São Paulo e no Rio de Janeiro. Foram fundados por Sérgio Serafim Del Grande e Robson Sampaio de Almeida, que trouxeram o esporte ao Brasil após retornarem de tratamentos de reabilitação em hospitais americanos, onde adquiriram o conhecimento do esporte em cadeira de rodas. ${ }^{1,14}$

A participação brasileira nos Jogos Paralímpicos aconteceu pela primeira vez em 1972, em Heidelberg, Alemanha. A modalidade foi a bocha, mas sem conquista de medalhas. Em 1976, nos Jogos de Toronto, Canadá, nessa mesma modalidade, Robson de Almeida e Luis Carlos Coutinho conquistam as duas primeiras medalhas paralímpicas (prata) para o Brasil. $^{13}$

\section{A CLASSIFICAÇÃO FUNCIONAL}

Os sistemas de classificação têm sido amplamente utilizados nos esportes, a fim de estabelecer um ponto de partida justo e igualitário para as competições. ${ }^{16}$

Na prática do esporte convencional, encontramos critérios de classificação que visam a aproximar os atletas segundo a sua condição motora e/ou biológica, como é o caso das 
categorias por idade (ex.: infantil, juvenil, adulto ou master) e por sexo. No esporte paralímpico, diversas modalidades fazem uso de um sistema de classificação, desenvolvido especificamente para a modalidade esportiva. A classificação é dita funcional pelo fato de os atletas serem avaliados em relação à sua funcionalidade em situação de jogo. ${ }^{8}$

A classificação visa a organizar os atletas em classes para que possam competir em condições de paridade funcional, conseqüentemente a competição torna-se mais justa, dessa forma permite que atletas com maiores comprometimentos físicos tenham oportunidade de participar de competições, assim como atletas que apresentam um menor grau de comprometimento. Para tal, é fundamental que haja um sistema de classificação eficiente e justo.

Para Guttmann ${ }^{17}$ o objetivo da classificação funcional no esporte paralímpico é: "assegurar a competição justa e eliminar as possibilidades de injustiças entre participantes de classes semelhantes e dar prioridade para as mais severas deficiências".

A classificação funcional no esporte paraolímpico tem vários objetivos: promover a igualdade na competição, promover a participação, estimular o envolvimento de um número considerável de classes e ajudar a distinguir e encorajar altos níveis de desempenho. ${ }^{18}$ Sendo a essência do esporte paralímpico com a necessidade de constante aprofundamento e evolução. ${ }^{9}$

O primeiro tipo de classificação para pessoas com deficiência física foi desenvolvido na Inglaterra, em 1940, por médicos e especialistas da área de reabilitação. Esta classificação é conhecida como classificação médica e foi desenvolvida para lesados medulares parciais ou totais, baseada no nível de lesão da medula. ${ }^{4,11,19}$

A classificação médica não perdurou por muito tempo, pois tinha muitos aspectos médicos e discordantes da prática esportiva, onde o atleta não utilizava seu verdadeiro potencial muscular. Este sistema se tornou incapaz de agrupar vários tipos de deficiência e funcionalidade, resultando em um número excessivo de classes. ${ }^{9}$ 
A partir disso a classificação funcional foi tema de discussão e estudo da tese de doutorado do professor Horst Strohkendel, de Colônia, Alemanha. Seu objetivo era propor um novo sistema de classificação funcional para o esporte paralímpico. As bases desse novo sistema de classificação proposto pelo médico alemão, contou com a ajuda de Bernard Courbariax (professor de Educação Física) e Phill Craven (jogador de basquete em cadeira de rodas). ${ }^{11}$

Este sistema revolucionou o esporte para deficientes em cadeira de rodas, já que permitia o ingresso de outras deficiências além da lesão medular na modalidade.

A classificação funcional não estava mais voltada para as limitações motoras dos atletas, mas sim no chamado "potencial residual" do atleta.

O método consiste em uma categorização, que o atleta recebe em função de seu volume de ação, ou seja, de sua capacidade de realizar movimentos, colocando em evidência a potencialidade motora dos resíduos musculares da sequiela de algum tipo de deficiência, bem como, os músculos que não foram lesados. $5,12,20-23$

A avaliação é realizada após uma análise do potencial de ação de cada atleta, incluindo avaliação de tronco, propulsão na cadeira de rodas, drible, arremesso e rebote, através de testes e avaliação em situação de jogo. ${ }^{19}$

Este sistema foi utilizado pela primeira vez em 1984, nos Jogos Mundiais para Deficientes na Inglaterra e aceito nos Jogos Paralímpicos de Seul, 1988. ${ }^{5,24}$

Devido à falta de aperfeiçoamento desse sistema, alguns problemas foram enfrentados pelo International Paralympic Commitee (IPC), como o cancelamento de eventos pela falta de competidores. Outros problemas encontrados foram a pouca competitividade em provas com número pequeno de participantes e provas com grande número de atletas causando uma demora excessiva para sua realização. ${ }^{4}$

Em função desses problemas o IPC exigiu que cada um de seus comitês esportivos desenvolvesse um sistema de classificação específico de cada esporte que diminuíssem 
significativamente o número das classes e que o sistema novo fosse executado a tempo para os Jogos Paralímpicos de 1992 em Barcelona. ${ }^{15,}$ 19, 23

O uso do sistema de classificação funcional reduziu o número de classes, centrando-se sobre a capacidade funcional em vez da deficiência, e acabou por conduzir a um aumento no número de eventos nas principais competições paralímpicas. ${ }^{21}$

Desde então as modalidades esportivas para pessoas com deficiência foram gradativamente absorvendo os princípios da classificação funcional e adaptando-a conforme a especificidade do esporte e habilidades funcionais de seus praticantes. ${ }^{25}$

\section{PROCEDIMENTOS DA CLASSIFICAÇÃO FUNCIONAL NOS DIFERENTES TIPOS DE DEFICIÊNCIAS}

Para cada modalidade, há um sistema próprio de classificação. Na maioria delas, esse sistema evoluiu muito nos últimos anos, deixando a perspectiva médica para a adoção da funcional. $^{4}$

Cada esporte determina seu próprio sistema de classificação baseado nas habilidades funcionais identificando as áreas chaves que afetam o desempenho para a performance básica do esporte escolhido. A habilidade funcional necessária independe do nível de habilidade ou treinamento adquirido. ${ }^{5,7,26-28}$

A classificação é um processo contínuo, quando um atleta começa a competir, eles são alocados em uma classe e pode ser revisto ao longo da carreira do atleta. As classes são determinadas por uma variedade de processos que incluem: avaliação médica (relacionada à especificidade da deficiência), funcional (relacionada à especificidade do esporte) e de observação dentro e fora de competição. ${ }^{23}$

A equipe de classificação deve ser composta por três profissionais da área de saúde: médico, fisioterapeuta e professor de Educação Física. A classificação é realizada em três estágios: médico, funcional e técnico. ${ }^{11,25}$ 
$\mathrm{Na}$ parte médica é feito um exame físico para verificar exatamente a patologia do atleta bem como sua inabilidade que afeta a função muscular necessária para um determinado movimento. Na avaliação funcional são realizados testes de força muscular, amplitude de movimento articular, mensuração de membros, coordenação motora, evidenciando os resíduos musculares utilizados para a performance na prova. ${ }^{29}$

Por último vem a avaliação técnica que consiste na demonstração da prova realizada utilizando as adaptações necessárias. São observados os grupos musculares na realização do movimento, técnica utilizada, também são observadas a utilização de próteses e órteses durante a prática desportiva. ${ }^{4}$

Durante a competição os classificadores poderão verificar o potencial funcional verdadeiro do atleta, algo que tenha ficado obscuro nos outros processos de classificação. Muitos esportes como o atletismo, basquete sobre rodas e a natação, têm a política que permite um classificador monitorar uma classificação por vários eventos. ${ }^{30}$

Tradicionalmente, há atletas que pertencem a seis grupos diferentes de deficiência no Movimento Paralímpico: paralisia cerebral, amputados, deficiência visual, lesões medulares, deficiência intelectual e um grupo que inclui todos aqueles que não se enquadram nos grupos acima referidos (les-autres). ${ }^{28}$

$\mathrm{Na}$ Paralisia Cerebral outros fatores neurológicos também se misturam e devem ser considerados no momento da classificação. Essa classificação depende do grau de espasticidade e de coordenação de cada atleta. É determinada pela CP - IRSA (Cerebral Palsy - International Sports and Recreation Association). ${ }^{21,31}$

Existem as classes CP1 a CP8, sendo que o comprometimento maior esta nas classes mais baixas e o esporte mais praticado por pessoas com Paralisia Cerebral é a Bocha Adaptada. ${ }^{1}$

Para os atletas com amputações e les-autres os sistemas de classificação são determinados pela ISOD (International Sports Organization for the Disabled). Para os atletas amputados existem nove classes funcionais e dois tipos de classificação: Uma de acordo com a altura da amputação e outra conforme o comprimento do membro amputado considerando o 
esporte praticado. ${ }^{11}$ Para atletas em condição de les-autres existem duas maneiras de classificação, o primeiro método é baseado no número de membros afetados e na complexidade da condição física. O outro método é baseado no movimento em si, ou seja, na função motora. ${ }^{32}$

Na Deficiência Visual a classificação é determinada pela International Blind Sports Association (IBSA), a classificação é realizada de acordo com os parâmetros funcionais da acuidade visual e do campo visual, e são classificados em: B1 - Cegueira Total, incapacitando o reconhecimento de objetos ou contornos; B2 - Limitação no campo visual em $5^{\circ}$ ou acuidade visual de 2/60; B3 - Limitação no campo visual entre $5^{\circ}$ e $60^{\circ}$ ou acuidade visual entre $2 / 60$ a $6 / 60 .^{4,11,31,33}$

Em algumas modalidades do esporte para deficientes visuais existem adaptações diferentes em relação a outras deficiências, tais como uso da venda por todos os atletas de Goalball, independente da classificação, no atletismo, alguns atletas podem fazer uso de um atleta guia, e no judô os atletas já iniciam na posição inicial da luta.

Atualmente a maioria dos desportos paraolímpicos utiliza sistemas de classificação funcional. Uma exceção notável é o sistema de classificação utilizado pelo (IBSA), que permanece no modo clínico. $^{28}$

Atletas com lesão medular, espinha bífida e poliomielite são classificados dentro da categoria de atletas que usam cadeira de rodas. Estes atletas estão dentro de oito classes baseadas no nível de lesão e do resultado encontrado na função muscular. ${ }^{7,25,34}$

Essa classificação inclui a análise da função muscular, que é medida e avaliada para cada lado do corpo. A permissão para competir depende da presença de no mínimo $10 \%$ de comprometimento na função. ${ }^{11,15,35}$

O esporte para pessoas com deficiência intelectual, internacionalmente é administrado por duas organizações: International Sports Federation for Person with Intellectual Disability (INAS-FID) e Special Olympics Inc. (SOI). Durante os Jogos Paralímpicos participam atletas com deficiências intelectuais leves e moderadas, competindo em uma classe única. 
Estes atletas foram os últimos a serem incluídos nesse evento e competem nas modalidades de natação, atletismo, tênis de mesa, basquetebol entre outras. ${ }^{1}$

Assim o ato de proporcionar igualdade em qualquer sistema de classificação funcional estabelece um menor número de classes, de acordo com a equidade de oportunidades para os atletas e isto sugere que deve haver diferenças significativas no desempenho entre as classes, juntamente com diferenças de desempenho pessoal de cada atleta no esporte paralímpico.

\section{CONSIDERAÇÕES FINAIS}

A classificação funcional é um dos itens mais importantes na prática esportiva, é responsável pela igualdade e a inclusão de diferentes níveis de deficiências dentro de competições esportivas.

Percebe-se que atualmente há uma grande evolução no conhecimento de métodos e procedimentos da classificação funcional. Com o crescente número de atletas, competições

e modalidades esportivas faz-se necessário e de fundamental importância o desenvolvimento dos métodos de avaliação na classificação funcional, já que esse processo é contínuo e exige conhecimentos cada vez mais aprofundados.

Assim os sistemas de classificação funcional nas diferentes modalidades do esporte paralímpico continuam em constante evolução e buscam aprimorar seus métodos afim de proporcionar a participação e a valorização do atleta com deficiência, dessa forma, garantindo que o nível de treinamento e a habilidade de cada um sejam os fatores decisivos para o sucesso durante as competições.

\section{REFERÊNCIAS}

${ }^{1}$ GORGATTI, M. G.; GORGATTI, T. O esporte para pessoas com necessidades especiais. In: ; DA COSTA, R. F. (Ed.). Atividade física adaptada: qualidade de vida para pessoas com necessidades especiais. Barueri: Manole, 2005. 
${ }^{2}$ BRASILE, F. M. Wheelchair basketball skills proficiencies versus disability classification. Adapted Physical Activity Quarterly, Champaign, v. 3, n.1, p. 6-13, 1986.

${ }^{3}$ CASTEllanO, M. L.; ARAÚJO, P. F. Avaliação a partir do volume de jogo para determinar a classificação em basquete sobre rodas. In: GORLA, J. I. Educação Física adaptada: o passo a passo da avaliação. São Paulo: Phorte, 2008.

${ }^{4}$ FREITAS, P. S. Fundamentos básicos da classificação funcional do esporte para deficientes físicos. Revista da Sociedade Brasileira de Atividade Motora Adaptada, Bauru, v.10, n. 1, p. 22-25, 2005.

${ }^{5}$ STROHKENDL, H. The 50th Anniversary of wheelchair basketball. New York: Waxmann, 1996.

${ }^{6}$ HOWE, P. D.; JONES, C. Classification of disabled athletes: (Dis)empowering the paralympic practice community. Sociology of Sport Journal, Champaign, v. 23, n. 1, p. 29-46, 2006.

${ }^{7}$ VANLANDEWIJCK, Y. C.; SPAEPEN, A. J.; LYSENS, R. J. Wheelchair propulsion efficiency: movement pattern adaptations to speed changes. Medicine and Science in Sports and Exercise, Hagerstown, v. 26, n. 1, p. 373-381, 1994.

${ }^{8}$ TWEEDY, S.; VANLANDEWIJCK, Y. C. International Paralympic Committee Position Stand - Background and scientific rationale for Classification in Paralympic Sport. British Journal of Sports Medicine, Loughborough, v. 10, n. 10, p. 37-48, 2009.

${ }^{9}$ SHERRIL, C. Disability sport and classification theory: A new era. Adapted Physical Activity Quarterly, Champaign, v. 16, n. 3, p. 206-215, 1999.

${ }^{10}$ ADAMS, R. C. et al. Jogos, esportes e exercícios para o deficiente físico. 3. ed. São Paulo: Manole, 1985. 
${ }^{11}$ MAUERBERG-DeCASTRO, E. Atividade Física adaptada. Ribeirão Preto: Teccmed, 2005 .

${ }^{12}$ MATTOS, E. Introdução à classificação funcional. Revista da Sociedade Brasileira de Atividade Motora Adaptada, Bauru, v. 4, n. 4, p. 11-13, 1998.

${ }^{13}$ ARAÚJO, P. F. Desporto adaptado no Brasil: origem, institucionalização e atualidades. 1997. Tese (Doutorado em Educação Física) - Faculdade de Educação Física, Universidade Estadual de Campinas, Campinas, São Paulo, 1997.

${ }^{14}$ CIDADE, R. E. A.; FREITAS, P. S. Introdução à Educação Física e ao desporto para pessoas portadoras de deficiência. Curitiba: Ed. da UFPR, 2002.

${ }^{15}$ WINNICK, J. P. Educação Física e esportes adaptados. 3. ed. Barueri: Manole, 2004.

${ }^{16}$ RITCHER, K. J. et al. Integrated swimming classification: a faulted system. Adapted Physical Activity Quarterly, Champaign, v. 9, n. 1, p. 5-13, 1992.

${ }^{17}$ GUTMANN, L. Textbook of sport for the disabled. Aylesbury: HM\&M, 1976.

${ }^{18}$ GOODMAN, S. Couching athletes with disabilities: general principles. Canberra: Australian Sports Commission,1993.

${ }^{19}$ COURBARIAUX, B. The Classification System for Wheelchair Basketball players. New York: IWBF, 1996.

${ }^{20}$ RITCHER, K. J. Integrated classification: an analysis. In: STEADWARD, R. D.; NELSON, E. R.; WHEELER, G. D. (Ed.). Vista 93: the outlook. Edmonton: Rick Hansen Centre, 1993. p. 255-259.

${ }^{21}$ VANLANDEWIJCK, Y. C.; CHAPPEL, R. J. Integration and classification issues in competitive sports for athletes with disabilities. Sport Science Review, Champaign, v. 5, n.1, p. 65-68, 1996. 
${ }^{22}$ BRASILE, F. M.; HEDRICK, B. N. The relationship of skills of elite wheelchair basketball competitors to the International functional classification system. Therapeutic Recreation Journal, v. 30, n. 2, p. 114-127, 1996.

${ }^{23}$ STROHKENDL, H. Implications of sports classification systems for persons with disabilities and consequences for science and research. In: DOLL-TEPPER, G.; KRONER, M.; SONNENSCHEIN, W. Vista'99: new horizons in sport for athletes with a disability. Koln: Meyer \& Meyer Sport, 2001. p. 281-301.

${ }^{24}$ BRASILE, F. M. Wheelchair sports: A new perspective on integration. Adapted Physical Activity Quarterly, Champaign, v. 7, n. 1, p. 3-11, 1990.

${ }^{25}$ VANLANDEWIJCK, Y. C. et al. Proportionality in wheelchair basketball classification. Adapted Physical Activity Quarterly, Champaign, v. 20, n. 4, p. 369-380, 2003.

${ }^{26}$ WEISS, M.; CURTIS, K. A. Controversies in medical classification of wheelchair athletes. In: SHERRIL, C. (Ed.). Sport and disabled athletes: 1984 Olympic Scientific Congress Proceedings. Champaign: Human Kinetics, 1996. p. 93-100.

${ }^{27}$ WU, S. K.; WILLIAMS, T. Paralympic swimming performance, impairment, and the functional classification system. Adapted Physical Activity Quarterly, Champaign, v. 16, p. 251-270, 1999.

${ }^{28}$ INTERNATIONAL PARALYMPIC COMMITTEE (IPC). The History of Classification. $2010 . \quad$ Disponível em: $<$ http://www.paralympic.org/export/sites/default/Sport/Classification/2010_04_22_x1x_Hi story.pdf >. Acesso em: 28 abr. 2013.

${ }^{29}$ DOYLE, T. L. A. et al. Further Evidence to change the Medical Classification System of The National Wheelchair Basketball Association. Adapted Physical Activity Quarterly, Champaign, v. 21, n. 1, p. 63-70, 2004. 
${ }^{30}$ GIL-AGUDO, A.; AMA-ESPINOSA, A. D.; CRESPO-RUIZ, B. Wheelchair Basketball Quantification. Physical Medicine and Rehabilitation Clinics of North America, Philadelphia, v. 21, n.1 p. 141-156, 2010.

${ }^{31}$ MATTOS, E.; GORGATTI, M. G. Avaliação em esporte para portadores de deficiência. In: KISS, M. A. P. D. M. (Ed.). Esporte e exercício: avaliação e prescrição: Roca Biomedicina, 2003.

${ }^{32}$ GEHLSEN, G. M.; KARPUK, J. Analysis of the NWAA swimming classification system. Adapted Physical Activity Quarterly, Chmapaign, v. 9, n. 1, p. 141-147, 1992.

${ }^{33}$ KHALILI, M. A. Quantitative sports and functional classification (QSFC) for disabled people with spasticity. British Journal of Sports Medicine, Loughborough, v. 38, p. 310313, 2004.

${ }^{34}$ SHENG, K. W.; WILLIAMS, T. Paralympic swimming performance, impairment and the functional classification system. Adapted Physical Activity Quarterly, Champaign, v. 16, n.1, p. 251-270, 1999.

${ }^{35}$ MOLIK, B. et al. Relationship between functional classification levels and anaerobic performance of wheelchair basketball athletes. Research Quarterly for Exercise and Sport, Reston, v. 81, n. 1, p. 69-73, 2010. 